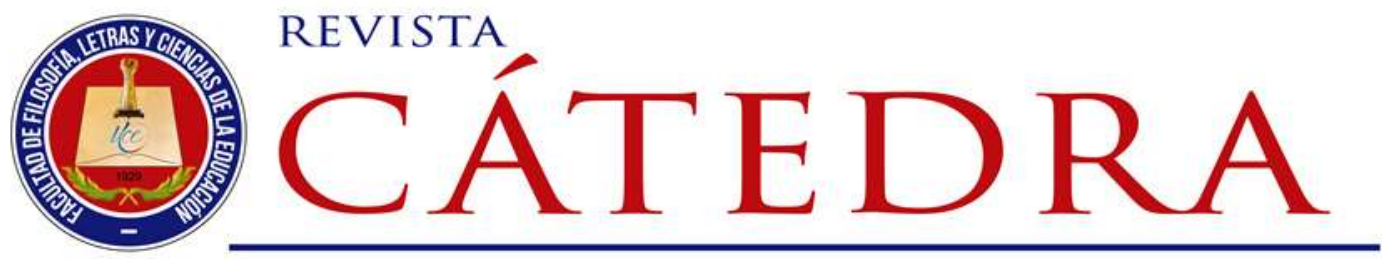

\title{
El enfoque de la educación virtual desde una perspectiva holístico frente a la pandemia del COVID - 19
}

\author{
The approach to virtual education from a holistic \\ perspective in the face of the COVID - 19 pandemic
}

\author{
Dina Inga-Lindo \\ Universidad Nacional Mayor de San Marcos, Lima, Perú \\ dingal@unmsm.edu.pe \\ https://orcid.org/0000-0001-6998-3592
}

Felipe Aguirre-Chávez

Universidad Nacional Mayor de San Marcos, Lima, Perú

caguirrech@unmsm.edu.pe https://orcid.org/0000-0002-5799-0653

(Recibido: 02/12/2020; Aceptado: 16/12/2020; Versión final recibida: 05/01/2021)

Cita del artículo: Inga, D. y Aguirre, F. (2021). El enfoque de la educación virtual desde una perspectiva holística frente a la pandemia del Covid-19. Revista Cátedra, 4(1), 81-97.

\section{Resumen}

A raíz de la pandemia COVID-19, que viene aquejando a la humanidad, se determinó en diversos países que las clases se desarrollen vía online, con el propósito de no perder el año académico, ni la masiva deserción de los estudiantes universitarios. El estudio emerge de la experiencia empírica y la revisión de variados documentos científicos que enfocan sus estudios desde una visión panorámica de las personas, con el fin de determinar en qué medida el enfoque de la educación virtual cumple con impartir un aprendizaje holístico en estudiantes universitarios. Los resultados fueron analizados estadísticamente indicando la confiabilidad del instrumento aplicado a cinco dimensiones con sus respectivos ítems, en un rango elevado de 0.933 , las pruebas resultaron satisfactorias. En cuanto al análisis descriptivo de las dimensiones resalta la categoría "casi siempre" con 67\%, aplicado al proceso de la enseñanza - aprendizaje virtual. Se concluye que la educación virtual que se 
imparte posee una visión holístico por los efectos positivos de satisfacción encontrados en los estudiantes universitarios. Cabe resaltar que es necesario utilizar las herramientas digitales de manera inteligente, multidimensional, flexible, con disciplina y que impulse el aprendizaje activo, tomando como base el sistema asincrónico y sincrónico.

\section{Palabras claves}

Aprendizaje holístico, COVID-19, enfoque virtual, innovación tecnológica, renovación educativa.

\section{Abstract}

As a result of the COVID-19 pandemic, which has been afflicting humanity, it was determined in several countries that classes should be held online, in order not to lose the academic year, nor the massive dropout of university students. The study emerges from the empirical experience and the review of various scientific documents that focus their studies from a panoramic vision of man, in order to determine to what extent the approach of virtual education complies with providing a holistic learning in university students. The results were analyzed statistically indicating the reliability of the instrument applied to five dimensions with their respective items, in a high range of 0.933 , the tests were satisfactory. As for the descriptive analysis of the dimensions, the category "almost always" stands out with $67 \%$, applied to the process of virtual teaching and learning. It is concluded that the virtual education that is given has a holistic vision due to the positive effects of satisfaction found in the university students. It is necessary to emphasize that it is necessary to use the digital tools in an intelligent, multidimensional, flexible way, with discipline and that it impels the active learning, taking as a base the asynchronous and synchronous system.

\section{Keywords}

Holistic learning, COVID-19, virtual approach, technological innovation, educational renewal.

\section{Introducción}

El impacto que causó la pandemia del COVID-19, representa un desafío en el avance de la educación como proceso y resultado. Según los datos obtenidos de la CEPAL-UNESCO (2020):
Esta emergencia dio lugar al cierre masivo
de las actividades presenciales de instituciones educativas en más de 190 países con el fin de evitar la propagación del virus y mitigar su impacto. Para el mes de mayo, más de 1.200 millones de estudiantes de todos los niveles de enseñanza, en el mundo, habían dejado de tener clases presenciales. De ellos, más de 160 millones eran estudiantes de América Latina y el Caribe (pág.1).

De acuerdo a lo señalado, muchos países buscaron diversas formas de enseñanza, con el deseo de que no se interrumpa las labores académicas, el cual los llevó a decidir por una educación virtual, que con el paso de los meses se convirtió en una nueva oportunidad, para el desarrollo del aprendizaje de los estudiantes. En este sentido "muchos colegios y universidades han cancelado las clases en el campus, requiriendo instrucción en línea para 
continuar, esto significa que la educación a distancia no es una consideración sino una necesidad" (Abreu, 2020, pág. 2).

El Perú no estuvo exento a este dilema, se buscó responder adecuadamente a la contingencia desde una perspectiva ingeniosa y rápida de las autoridades del Ministerio de Educación, con el propósito de crear mecanismos que aseguren resultados positivos en la continuidad escolar, mitigando la deserción y acortando las brechas de la desigualdad educativa, que el país atraviesa desde hace décadas. A pesar que el gobierno tomó medidas preventivas desde el inicio de la pandemia, los efectos no son nada alentadores en la economía, la educación y la salud. No obstante, el cambio en educación dio un giro de $180^{\circ}$ en las prácticas educativas, empezando por transformar la mirada docente, desde el punto de vista cognitiva, metodológica, tecnológica y emocional. Se asumió el reto y en el proceso se fue aprendiendo el manejo de las herramientas digitales, a pesar de las circunstancias adversas, se priorizaron el aprendizaje significativo, y el desarrollo de habilidades mediante diversos mecanismos en las plataformas e-learning. Las capacitaciones y la , búsqueda de tutoriales en YouTube fueron necesarias, para aprender rápido el teje y desteje de las estrategias y herramientas tecnológicas de G Suite; Classroom, Meet, Gmail, Hangouts, Calendar, Drive, Sites, Forms etc., sobre el cual manifiesta Sotelo (2017)“Las computadoras e Internet están al alcance de todos (niños, jóvenes y adultos), aprender a utilizarlos y sacar provecho de estos es cuestión de actitud que, pasa por superar el miedo a la tecnología y tener disposición para aprender" (pág. 40), a pesar de las circunstancias adversas, se dio el paso de la educación convencional a una virtual, utilizando la tecnología digital, para transformar el aprendizaje de los estudiantes por medio de la comunicación sincrónica, (Webconference, chat), asincrónica (plataformas, aplicaciones tecnológicas, recursos digitales y retroalimentación o feedback) entre el docente y estudiante dentro de la interconectividad.

En el caso de los estudiantes, la pandemia trajo consigo la vulnerabilidad socioeconómica, acentuando el riesgo de la desigualdad y la deserción, en aquellos jóvenes que menos recursos tienen, por ejemplo, el desempleo de sus padres, la desnutrición, la conectividad o acceso a internet y los cambios cognitivos, psicológicos, culturales que sufrieron para incorporarse al aprendizaje $100 \%$ online, a pesar del manejo tecnológico de algunas herramientas que los estudiantes poseían, es "una situación particular que ha de atenderse es el riesgo de desvinculación educativa y abandono escolar de estos grupos más vulnerables a los efectos de la pandemia y la consecuente crisis sanitaria, social y económica" (UNESCO 2020, pág. 15). Del panorama anteriormente se desprende, el problema general de la investigación, ¿En qué medida el enfoque de la educación virtual cumple con impartir un aprendizaje holístico en estudiantes universitarios? Tomando como punto de vista lo indicado por Gluyas et al., (2015):

La formación holística requiere de la integración de saberes: saber ser a partir del autoconocimiento, para proyectarlo en un saber conocer que motive el aprendizaje continuo con miras a ser reflejado en un saber hacer que impacte en el desarrollo del entorno inmediato, con resonancia en la sociedad y en la humanidad (pág. 3).

¿Los estudiantes universitarios serán educados de manera holístico? Cubas (entrevistado, 2013) manifiesta:

Hay escasez de talento en un 88\%, debido a la falta de un plan estratégico en educación y a la inadecuada preparación profesional que reciben en 
las universidades para enfrentarse al mundo laboral. La experiencia empírica demuestra que a pesar que la enseñanza sea presencial o virtual no se cumple con el objetivo estratégico de calidad y excelencia educativa que tanto añora el país (pág. 5).

El objetivo general de la investigación es determinar en qué medida el enfoque de la educación virtual cumple con impartir un aprendizaje holístico que se optó frente a la Pandemia del COVID -19 en estudiantes universitarios, del objetivo general se desglosa en los siguientes objetivos específicos: a. Determinar la confiabilidad del instrumento (cuestionario) para medir el enfoque de la educación virtual desde una perspectiva holístico frente a la pandemia del COVID - 19. b. Valorar el nivel de impacto que ocasiono la COVID 19 en el enfoque de una educación virtual. c. Valorar el nivel de aprendizaje de los estudiantes tras la implementación de la educación virtual que se optó frente a la Pandemia del COVID -19en estudiantes universitarios.

El propósito del estudio es poner en relieve, desde una perspectiva real y objetiva, si las clases impartidas de manera no presencial en el nuevo contexto virtual favorecen al desarrollo integral de los estudiantes universitarios, teniendo en cuenta que la labor docente debe perfilar estudiantes idóneos, capaces de lograr aprendizajes significativos desde un enfoque holístico y construir conocimientos más complejos que exige el adelanto globalizado de la ciencia y la tecnología.

El presente trabajo está estructurado de la siguiente manera: revisión de la literatura, donde se estipula las definiciones de las variables con argumentos de autoridad; materiales y métodos, está orientado a plasmar el enfoque de estudio, el diseño de investigación, población y muestra, instrumento de recolección de información; elementos flotantes, está orientado a la validez y confiabilidad del instrumento, análisis descriptivo, resultados; discusión de los resultados. Para dar cierre a la investigación se determina las conclusiones y referencias.

\section{Revisión de la literatura}

En el 2020 el mundo fue evidente del surgimiento de una pandemia inimaginable, cuya letalidad en los primeros meses, se convirtió en una amenaza natural que afloraba la extinción del hombre, causando pánico por la rápida propagación y que puso en tela de juicio a la población; la OMS, observando el avance voraz declara a la COVID 19 como pandemia. En el Perú se detectó el primer caso el 15 de marzo por el cual el presidente Martín Vizcarra y sus ministros determinaron el aislamiento social obligatorio (cuarentena), empezando por el cierre de fronteras y el estricto cumplimiento de los protocolos de salubridad impuestas por el Ministerio de Salud, con el propósito de paliar la propagación y las decenas de muertes por día. Al respecto Velazque et al., (2020) menciona:

El COVID-19 ha cambiado la vida de más de 33millones de peruanos, así como también provocó una crisis en la educación superior, cerrando sus puertas el $87 \%$, las clases de ciclos vacacional fueron interrumpidas, los estudiantes y docentes que cursaban pasantías e intercambios a nivel nacional e internacional fueron cancelados (pág. 204).

La SUNEDU y el Ministerio de Educación tomaron la decisión de que las clases se llevarían a cabo de manera no presencial, utilizando las diversas plataformas, rompiendo así la enseñanza tradicional y logrando la adaptabilidad de los estudiantes para recibir sus clases de manera virtual y no perder el año académico. Frente a ello Cardini et al., (2020) afirma: 
Esta implementación, traerá consigo grandes desafíos, que se debe enfrentar, para garantizar la continuidad del proceso pedagógico. Como la distribución del contenido temático, a través de las plataformas digitales, ampliación del acceso a la tecnología, el acompañamiento y fortalecimiento de capacidades de los docentes en el contexto de aislamiento y las capacitaciones en el uso de tecnologías digitales (pág.8).

La renovación es necesaria para comprender la realidad, convivir y actuar en tiempos de crisis, ya sea de manera individual o en equipo para enfrentar sin temor al nuevo contexto, frente a ello "la educación virtual utiliza estrategias de enseñanza - aprendizaje que permiten superar las limitaciones de espacio y tiempo entre los actores del proceso educativo" (Gallego, 2013, pág. 157), la decisión fue acertada, sabiendo que la educación no debe parar, porque con ello se busca el empoderamiento de los jóvenes para apropiarse del abanico de conocimientos y construir su realidad con bases sólidas, impulsando el desarrollo de las habilidades blandas y duras que permiten identificar y afrontar desafíos que encuentran en el trascurso de su vida personal, profesional y social. Al respecto la UNESCO manifiesta que en estas clases no presenciales se debe proyectar medidas para la continuidad, equidad e inclusión, centradas en las poblaciones vulnerables y marginadas; buscar la calidad y pertinencia de los contenidos y programas. Así como también el apoyo especializado al personal docente, asegurando condiciones adecuadas para cumplir su labor idónea en la educación a distancia. Al respecto, Allen y Seaman (2017) señala:

En el informe anual del estado de la educación en línea en los Estados Unidos, apuntan que el 35,6\% de los líderes académicos calificaron los resultados de aprendizaje en la educación mixta, blended o semipresencial como similares o superiores a los de la enseñanza cara a cara. El 63,3\% de esos líderes consideran a la educación en línea como fundamental para la estrategia institucional a largo plazo, frente al 13,7\% que considera que no, (pág.16-39).

En las clases virtuales, el rendimiento de los estudiantes depende del diseño pedagógico formativo, las herramientas digitales, estrategias, recursos que son necesarios para potenciar el aprendizaje activo, con el precepto de encaminar al buen desarrollo conceptual, procedimental y actitudinal de los jóvenes universitarios. Según Durall, et al., (2012):

Las principales tendencias son: primero, las personas pretenden poder trabajar, aprender y estudiar cuando quieran y desde donde quieran, segundo, la multitud de recursos y relaciones disponibles en Internet obligan a los educadores a revisar su rol en los procesos de creación de sentido, asesoramiento y acreditación, tercero, las tecnologías que se utilizan están, cada vez más, basadas en la computación en nube, y las nociones de apoyo a las tecnologías de información y comunicaciones están descentralizadas, cuarto, los cambios en la enseñanza universitaria inducen a la mayoría de las universidades a situar la capacitación de los docentes como un elemento estratégico en la calidad de la docencia y finalmente, el ámbito laboral es cada vez más colaborativo, lo que conlleva cambios en el modo de estructurar los proyectos estudiantiles (pág. 2).

La educación no presencial debe impartir un aprendizaje holístico, visto desde una concepción multidimensional donde todo conocimiento está concatenado, nada está 
aislado, todos se necesitan para promover el desarrollo y bienestar de la humanidad, al respecto Gallegos (1999) menciona:

El alumno es un ser humano total (corporal, afectivo, cognitivo, social, estético y espiritual) inserto en un proyecto social, perteneciente a una comunidad (familia, escuela) que interactúa con la sociedad, tanto emocional como ideológicamente, y no solo es un cerebro receptor de información (pág. 42).

Así como también se debe "considerar a la educación, más que un acto de transmisión, repetición y memorización de conocimientos, e incorporar al proceso holística, en el cual se intercambian saberes, se develan experiencias en una comunicación bidireccional entre docentes y estudiantes" (López, 2018, pág. 315), de acuerdo a lo estipulado las universidades tienen el reto de impartir una educación de calidad a pesar de las circunstancias adversas que se enfrenta, donde el docente se convierte en guía estimulante que cultiva un aprendizaje holístico que genera conocimientos y comprende la nueva forma de enseñanza, con participación, creatividad, expresividad, manejo del pensamiento complejo, para luego integrarlo en un solo sistema, sustentado en una visión panorámica de los problemas de la educación y teniendo como precedente que "la holística es un fenómeno psicológico y social, enraizado en las distintas disciplinas humanas y orientada hacia la búsqueda de una cosmovisión basada en preceptos comunes al género humano" (Briceño et al., 2010, pág. 74), así como también provocar la trasformación del individuo en su estructura académica de modo integral, introduciendo lo físico, mental, emocional, historia, cultura, política e ideología etc. y que busque valorar la vida práctica, la ciencia, el sentimiento, el raciocinio, la intuición, la sensibilidad, pensando que cada situación que enfrenta es una ocasión para aprender, desaprender y reaprender. Para ello los docentes deben empoderarse de las herramientas digitales, partiendo de la utilización del sistema sincrónico, esto le ayudará a estimular la interacción, participación activa, mediante la videoconferencia de Google - Meet (Chat, videos, infografías etc.), y estará conectado al mismo tiempo, independiente del lugar o la hora con sus pupilos, y el sistema asincrónico; le ayudará a gestionar el contenido mediante el uso de las plataformas tecnológicas, (Documentos, foro, tareas, lecciones, ejercicios, enlaces etc.), si se cumple un estricto seguimiento , acompañamiento, asesoría y retroalimentación, se estará fomentando un aprendizaje holístico, tomando como bastión a la educación virtual.

\section{Materiales y Métodos}

Metodológicamente el estudio corresponde al enfoque cuantitativo sustentado en los principios epistemológicos del paradigma empírico-analítico y se centra en hechos y fenómenos sociales con escaso o nulo interés de la subjetividad de los investigadores, de igual forma, utiliza el cuestionario para la recolección de datos y las respuestas fueron analizadas estadísticamente.

El diseño del estudio es no experimental transeccional correlacional-causal porque describe las relaciones categoriales y las variables en los tiempos de pandemia del COVID 19 (Hernández et al., 2010, p. 217). Asimismo el estudio se realizó en un contexto concreto y los datos fueron recolectados en un único momento o tiempo.

La población estuvo constituida por 720 estudiantes y la muestra fue de 100 estudiantes universitarios matriculados a las clases no presenciales 2020 -I, pertenecientes a un mismo ciclo académico de pregrado, que oscilan entre 18 a 22 años de edad, 62 varones y 38 mujeres respectivamente. 
El instrumento empleado fue una encuesta diseñada, en Google Forms, con la finalidad de proporcionar información relevante sobre el nuevo enfoque educativo virtual desde una perspectiva holística, que se viene desarrollándose a causa de la pandemia del COVID- 19 en los estudiantes universitarios. La constitución del instrumento fue de 05 dimensiones: 1. Utilización de las herramientas tecnológicas con 08 ítems, 2. Estrategia docente en la enseñanza virtual con 05 ítems, 3. Calidad formativa de la asignatura con 04 ítems, 4. Trabajo individual y en equipo con 04 ítems y 5 . Actitudes y valores frente a las clases virtuales con 3 ítems. Las respuestas se recogieron mediante escalas de Likert, donde; 1. Nunca, 2. Casi nunca, 3. A veces, 4. Casi siempre y 5. Siempre. Las características del cuestionario se obtuvieron mediante distintos tipos de análisis estadísticos realizados con la ayuda del programa IBM SPSS.

La técnica de recolección de datos fue a través del formulario en Google donde se hilvanó 24 preguntas de tipo Likert, la forma de evaluar fue de sección múltiple con respuestas cerradas y se configuraron para que los estudiantes respondieran de form a obligatoria. En las instrucciones se dio a conocer las razones de la encuesta y el tiempo que puede tomar desarrollar dicho instrumento. El cuestionario fue enviado a los estudiantes por medio del correo electrónico, luego se efectuó el análisis y la transcripción de la información según el interés de la investigación.

\section{Elementos flotantes}

Los resultados de la investigación muestran la validación del instrumento y sobre el enfoque de la educación virtual desde una perspectiva holística frente a la pandemia del COVID - 19 y los resultados descriptivos e inferenciales del estudio.

\subsection{Análisis de confiabilidad}

La evaluación de confiabilidad de un cuestionario conlleva la realización del análisis de consistencia interna. Para ello se calculó el coeficiente alfa de Cronbach, considerándose la siguiente categorización del coeficiente de confiabilidad: 0,8- 1 (elevado), 0,6-0,8 (aceptable), 0,4-0,6 (regular), 0,2-0,4 (bajo) y menor de 0,2, muy bajo (23).

De acuerdo al cuadro 1, se realizó un análisis de fiabilidad para cada dimensión o factor. En el proceso no se eliminó ningún ítems, el alfa general se encuentra dentro del rango elevado con 0.933 . En conclusión, se obtuvo un instrumento válido y fiable para el cuestionario del enfoque de la educación virtual desde una perspectiva holística frente a la pandemia del COVID -19 .

TIE5 Señala en qué grado las exposiciones y debates en las clases virtuales han contribuido en tu aprendizaje o despertaron tu interés.

AVDCO1 El nuevo contexto educativo ha cambiado mi visión sobre el papel del estudiante universitario

AVDCO5 La educación virtual ha cambiado mi actitud, en la manera de afrontar mis Dimensión estudios

1 AVDCO2 He asumido responsabilidades en el proceso de aprendizaje virtual, porque me permite trabajar a mi ritmo.

TIE1 Señala en qué grado las tareas han contribuido en tu aprendizaje a lo largo de la clase online.

UHT8 Las clases no presenciales te incentivaron al autoaprendizaje. 


\begin{abstract}
TIE2 Consulto otro material, aparte de lo presentado por el docente, para profundizar mi conocimiento.

DREE5 Si tuviera que describir la "sensación de cercanía y capacidad motivadora" del docente la calificaría como:

UHT10 las clases online facilitaron la transferencia de conocimientos

UHT9 Permiten el acceso a mayor información para aumentar mis conocimientos

UHT11 Los materiales utilizados en las clases ofrecieron una mejor presentación de los

Dimensión contenidos

2

UHT7 Las clases online te facilitan el recuerdo de la información y refuerzan los contenidos de aprendizaje.

DREE3 La accesibilidad a los profesores (disponibilidad, tiempo que tardaban en responder las consultas, calidad del feedback...) ha sido

DREE4 Los docentes adecuaron sus estrategias didácticas a las condiciones en que se desarrolla la asignatura (horario, secuencia, temporalización, espacio, material, etc.)

Dimensión

DREE2 ¿El docente se enfoca principalmente a la enseñanza académica?

3

CFACV1 Has encontrado el curso formativamente estimulante

CFACV2 ¿Las actividades son direccionadas en aprendizaje social y emocional para desarrollar las habilidades blandas?

CFACV4 Los temas de la nueva información o problema se relacionan con los que sabía anteriormente.

Dimensión

4

UHT5 Las clases virtuales te facilitaron el trabajo en equipo

CFACV5 Las actividades planteadas me hacen desarrollar otras destrezas cognitivas (análisis, síntesis, crítica...) en el estudio

UHT13 Aumenta la relación con los compañeros y mejora la relación con los docentes.

UHT14 ¿Te has sentido "parte integrante" de una comunidad virtual de aprendizaje cooperativo con los profesores y compañeros, a pesar de la distancia?

Dimensión UHT6 Las clases virtuales te motivan a continuar aprendiendo.

5

UHT2 Accedía rápidamente al sistema sincrónico como videoconferencia, chat. Para recibir su clase online.

Cuadro 1. Distribución de ítems según dimensiones

De acuerdo al cuadro 2, se analizaron todas las dimensiones citadas y se observa un rango aceptable en todos los casos. Se pretende confirmar el número de dimensiones que mejor se adapte a este modelo, y la extracción no se realizó para un número fijo de factores, sino para todos aquellos cuyos autovalores sean mayor que 1, además de evaluar la comunalidad y que al menos 2 ítems estén dentro de un solo factor, siendo solo 5 dimensiones en este caso que explican el $65.07 \%$ de la varianza. Además, se ha seleccionado un método de rotación (Varimax).
\end{abstract}

Dimensión Alfa de Cronbach $\quad$ Elementos




\begin{tabular}{lll}
\hline General & 0.933 & 24 \\
Dimensión 1 & 0.885 & 8 \\
Dimensión 2 & 0.855 & 5 \\
Dimensión 3 & 0.789 & 4 \\
Dimensión 4 & 0.796 & 4 \\
Dimensión 5 & 0.618 & 3 \\
\hline
\end{tabular}

Cuadro 2. Resumen del análisis de fiabilidad

\subsection{Validación cuantitativa - validación de constructo}

Finalizado el proceso de análisis de confiabilidad, el siguiente paso fue determinar el número óptimo de dimensiones mediante un análisis factorial.

De acuerdo al cuadro 3, el valor de la medida de adecuación muestral KMO es de 0.889 (\&gt; 0.5). Además, el valor del nivel de significación de la prueba de esfericidad de Bartlett es 4.406e-7 aproximadamente 0 . Por tanto, tiene sentido realizar un análisis factorial.

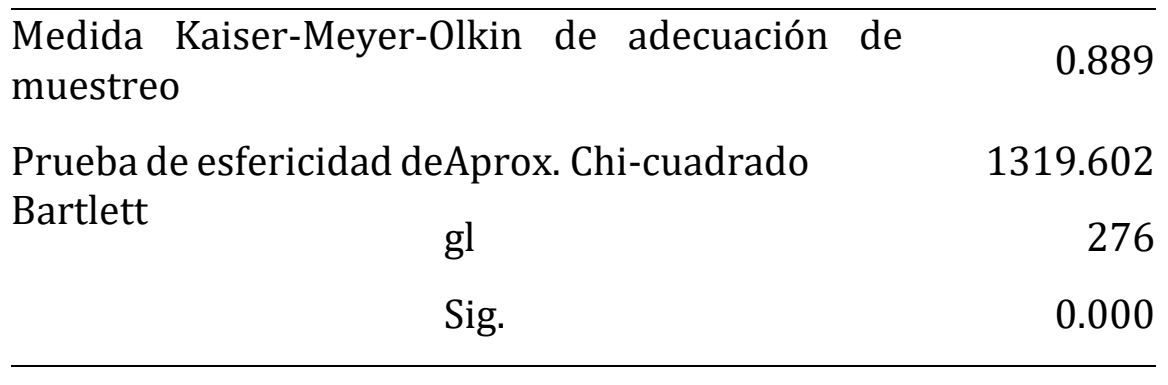

Cuadro 3. Prueba de Kmo y Bartlett

\subsection{Análisis descriptivo}

Una vez analizada la validación del constructo y la matriz de componentes rotados, es necesario analizar cada dimensión con sus respectivos ítems para evaluar el porcentaje que determine en qué medida el enfoque de la educación virtual cumple con impartir un aprendizaje holístico que se optó frente a la Pandemia del COVID -19en estudiantes universitarios. Para ello se muestra las cinco dimensiones: 1.Utilización de las herramientas tecnológicas con 08 ítems, 2. Estrategia docente en la enseñanza virtual con 05 ítems, 3. Calidad formativa de la asignatura con 04 ítems, 4. Trabajo individual y en equipo con 04 ítems y 5 . Actitudes y valores frente a las clases virtuales con 3 ítems. Conformados por las alternativas: 1 . Nunca, 2 . Casi nunca, 3 . A veces, 4 . Casi siempre y 5 . Siempre.

De acuerdo al cuadro 4, se puede observar que las preguntas de la dimensión 1 presentan porcentajes altos en "A veces" y "Casi Siempre" con valores superiores al 40\%. Destaca el ítem: "las clases no presenciales te incentivaron al autoaprendizaje" con un 29\% en "Siempre". Es necesario analizar cada dimensión con sus respectivas preguntas para evaluar el porcentaje de frecuencia.

\begin{tabular}{lccccc}
\hline Ítems & Nunca & Casi & A & Casi & \\
& & nunca & veces & siempre & Siempre
\end{tabular}


F1_AVDC01 El nuevo contexto educativo ha cambiado mi visión sobre el papel del $1 \% \quad 4 \% \quad 35 \% \quad 43 \% \quad 17 \%$ estudiante universitario

F1_AVDCO2 He asumido responsabilidades en el proceso de aprendizaje virtual, porque $\quad \begin{array}{llll}1 \% & 3 \% & 35 \% & 37 \%\end{array}$ me permite trabajar a mi ritmo.

F1_AVDC05 La educación virtual ha cambiado mi actitud, en la manera de $0 \% \quad 8 \% \quad 27 \% \quad 45 \% \quad 20 \%$ afrontar mis estudios

F1_DREE5 Si tuviera que describir la "sensación de cercanía y capacidad 1\% $\quad 5 \% \quad 27 \% \quad 46 \% \quad 21 \%$ motivadora" del docente la calificaría como:

F1_TIE1 Señala en qué grado las tareas han contribuido en tu aprendizaje a lo largo de la $\quad 1 \% \quad 4 \% \quad 32 \% \quad 44 \% \quad 19 \%$ clase online.

F1_TIE2 Consulto otro material, aparte de lo presentado por el docente, para profundizar $\quad 0 \% \quad 3 \% \quad 30 \% \quad 44 \% \quad 23 \%$ mi conocimiento.

F1_TIE5 Señala en qué grado las exposiciones $\mathrm{y}$ debates en las clases virtuales han contribuido en tu aprendizaje o despertaron $3 \%$ tu interés.

F1_UHT8 Las clases no presenciales te incentivaron al autoaprendizaje.

Cuadro 4. Utilización de las herramientas tecnológicas

De acuerdo al cuadro 5, se puede observar que las preguntas de la dimensión 2 destaca "la accesibilidad a los profesores (disponibilidad, tiempo que tardaban en responder las consultas, calidad del feedback...)" con un 58\% en "Casi siempre", y el ítem "Permiten el acceso a mayor información para aumentar mis conocimientos" con un $28 \%$ en "Siempre".

\begin{tabular}{|c|c|c|c|c|c|}
\hline ítems & Nunca & $\begin{array}{l}\text { Casi } \\
\text { nunca }\end{array}$ & $\begin{array}{l}\text { A } \\
\text { veces }\end{array}$ & $\begin{array}{l}\text { Casi } \\
\text { siempre }\end{array}$ & Siempre \\
\hline $\begin{array}{l}\text { F2_DREE3 La accesibilidad a los profesores } \\
\text { (disponibilidad, tiempo que tardaban en } \\
\text { responder las consultas, calidad del } \\
\text { feedback...) ha sido. }\end{array}$ & $1 \%$ & $3 \%$ & $20 \%$ & $58 \%$ & $18 \%$ \\
\hline $\begin{array}{l}\text { F2_UHT7 Las clases online te facilitan el } \\
\text { recuerdo de la información y refuerzan los } \\
\text { contenidos de aprendizaje. }\end{array}$ & $2 \%$ & $12 \%$ & $38 \%$ & $35 \%$ & $13 \%$ \\
\hline
\end{tabular}


F2_UHT9 Permiten el acceso a mayor información para aumentar mis $0 \% \quad 2 \% \quad 27 \% \quad 43 \% \quad 28 \%$ conocimientos.

F2_UHT10 las clases online facilitaron la transferencia de conocimientos.

$1 \% \quad 7 \% \quad 40 \% \quad 38 \% \quad 14 \%$

F2_UHT11 Los materiales utilizados en las clases ofrecieron una mejor presentación de los contenidos.

$1 \% \quad 0 \% \quad 34 \% \quad 43 \% \quad 22 \%$

Cuadro 5. Estrategia docente en la enseñanza virtual

De acuerdo al cuadro 6, se puede observar que las preguntas de la dimensión 3 resalta el ítem "Los docentes adecuaron sus estrategias didácticas a las condiciones en que se desarrolla la asignatura (horario, secuencia, temporalización, espacio, material, etc.)" con un valor del 58\% en "Casi siempre". Así como también el ítem "Los docentes adecuaron sus estrategias didácticas a las condiciones en que se desarrolla la asignatura (horario, secuencia, temporalización, espacio, material, etc.)" con un 58\% en "Casi siempre"

\begin{tabular}{|c|c|c|c|c|c|}
\hline Ítems & Nunca & $\begin{array}{l}\text { Casi } \\
\text { nunca }\end{array}$ & $\begin{array}{l}\text { A } \\
\text { veces }\end{array}$ & $\begin{array}{l}\text { Casi } \\
\text { siempre }\end{array}$ & Siempre \\
\hline $\begin{array}{llll}\text { F3_CFACV1 Has encontrado el curso } \\
\text { formativamente estimulante }\end{array}$ & $0 \%$ & $5 \%$ & $37 \%$ & $40 \%$ & $18 \%$ \\
\hline $\begin{array}{l}\text { F3_CFACV2 ¿Las actividades son direccionadas } \\
\text { en aprendizaje social y emocional para } \\
\text { desarrollar las habilidades blandas? }\end{array}$ & $3 \%$ & $4 \%$ & $34 \%$ & $44 \%$ & $15 \%$ \\
\hline $\begin{array}{l}\text { F3_DREE } 2 \text { ¿El docente se enfoca principalmente } \\
\text { a la enseñanza académica? }\end{array}$ & $0 \%$ & $1 \%$ & $8 \%$ & $67 \%$ & $24 \%$ \\
\hline $\begin{array}{l}\text { F3_DREE4 Los docentes adecuaron sus } \\
\text { estrategias didácticas a las condiciones en que } \\
\text { se desarrolla la asignatura (horario, secuencia, } \\
\text { temporalización, espacio, material, etc.) }\end{array}$ & $0 \%$ & $3 \%$ & $19 \%$ & $58 \%$ & $20 \%$ \\
\hline
\end{tabular}

Cuadro 6. Calidad formativa de la asignatura

De acuerdo al cuadro 7, se puede observar que las preguntas de la dimensión 4 presentan valores altos en Casi siempre, como ejemplos los ítems "Los temas de la nueva información o problema se relacionan con los que sabía anteriormente" y "Las actividades planteadas me hacen desarrollar otras destrezas cognitivas (análisis, síntesis, crítica...) en el estudio". Además se observa que el ítem "Aumenta la relación con los compañeros y mejora la relación con los docentes" tiene un valor del 56\% en la categoría de "A veces".

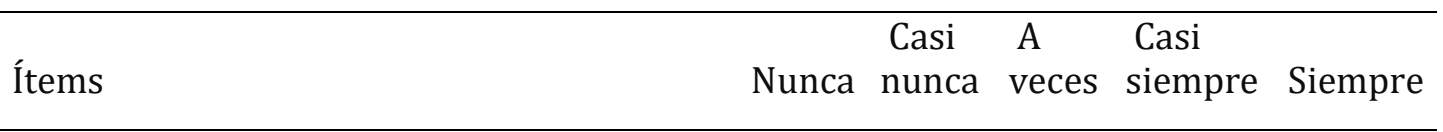


F4_CFACV4 Los temas de la nueva información o problema se relacionan con 2\% los que sabía anteriormente.

$0 \% \quad 30 \% \quad 60 \% \quad 8 \%$

F4_CFACV5 Las actividades planteadas me hacen desarrollar otras destrezas cognitivas (análisis, síntesis, crítica...) en el estudio $0 \%$

$3 \% \quad 17 \% \quad 59 \% \quad 21 \%$

F4_UHT5 Las clases virtuales te facilitaron el trabajo en equipo

$0 \% \quad 12 \% \quad 39 \% \quad 39 \% \quad 10 \%$

F4_UHT13 Aumenta la relación con los compañeros y mejora la relación con los $1 \% \quad 14 \% \quad 56 \% \quad 24 \% \quad 5 \%$ docentes.

\section{Cuadro 7. Trabajo individual y en equipo}

De acuerdo al cuadro 8, se puede observar que las preguntas de la dimensión 5 presenta un 63\% de "Casi siempre" en el ítem "Accedía rápidamente al sistema sincrónico como videoconferencia, chat" para recibir su clase online, sin embargo se observa un valor alto en la categoría "A veces" con un 44\%, además el ultimo ítem ¿Te has sentido "parte integrante" de una comunidad virtual de aprendizaje cooperativo con los profesores y compañeros, a pesar de la distancia?" Tiene un 40\% y 45\% en "A veces" y "Casi siempre".

\begin{tabular}{|c|c|c|c|c|c|}
\hline Ítems & Nunca & $\begin{array}{l}\text { Casi } \\
\text { nunca }\end{array}$ & $\begin{array}{l}\text { A } \\
\text { veces }\end{array}$ & $\begin{array}{l}\text { Casi } \\
\text { siempre }\end{array}$ & Siempre \\
\hline $\begin{array}{l}\text { F5_UHT2 Accedía rápidamente al sistema } \\
\text { sincrónico como videoconferencia, chat. } \\
\text { Para recibir su clase online. }\end{array}$ & $0 \%$ & $1 \%$ & $17 \%$ & $63 \%$ & $19 \%$ \\
\hline $\begin{array}{l}\text { F5_UHT6 Las clases virtuales te motivan a } \\
\text { continuar aprendiendo. }\end{array}$ & $1 \%$ & $16 \%$ & $44 \%$ & $29 \%$ & $10 \%$ \\
\hline $\begin{array}{l}\text { F5_UHT14 ¿Te has sentido "parte } \\
\text { integrante" de una comunidad virtual de } \\
\text { aprendizaje cooperativo con los profesores y } \\
\text { compañeros, a pesar de la distancia? }\end{array}$ & $2 \%$ & $5 \%$ & $40 \%$ & $45 \%$ & $8 \%$ \\
\hline
\end{tabular}

Cuadro 8. Actitudes y valores frente a las clases virtuales

\section{Discusión}

Los resultados de la investigación, demuestran que el enfoque de la educación virtual cumple con impartir un aprendizaje holístico que se optó frente a la Pandemia del COVID 19 en estudiantes universitarios. La UNESCO ha destacado desde finales de la década pasada, que la educación debe ser integral para cubrir todos los aspectos de la vida, con conocimientos científicos (aprender a conocer), destrezas profesionales (aprender a hacer), valores humanos y principios (aprender a ser), y el ejercicio de la responsabilidad ciudadana (aprender a convivir). Tomando como base esta idea, se elaboró las dimensiones del instrumento (encuesta) que fueron: utilización de las herramientas tecnológicas, (aprender a conocer), estrategia docente en la enseñanza virtual (aprender a convivir), calidad formativa de la asignatura (aprende a conocer), trabajo individual y en equipo 
(aprender hacer), actitudes y valores frente a las clases virtuales (aprender a ser), con el fin de proporcionar información fidedigna del tema expuesto en la investigación e impulsar en el estudiante universitario, el actuar de manera asertiva, autónomo, crítica y creativa, dejando traslucir sus habilidades y potencialidades para incorporarse al brazo cambiante de la sociedad.

En conclusión, se realizó un análisis de fiabilidad para cada dimensión o factor; en el proceso no se eliminó ningún ítem, el alfa general se encuentra en un rango elevado de 0.933.

Para el cuestionario del enfoque de la educación virtual desde una perspectiva holístico frente a la pandemia del COVID - 19, en el análisis de fiabilidad, se encontró el ítem UHT2 (Accedía rápidamente al sistema sincrónico como videoconferencia, chat, para recibir su clase online.) con una correlación elemento-total corregida menor a 0,4. Por tanto se sugiere eliminar.

Dentro del análisis descriptivo se encuentra la dimensión 1 que muestra valores altos en las categorías "A veces" y "Casi siempre"; le sigue la dimensión 2 con un comportamiento similar. En la dimensión 3 el ítem "¿El docente se enfoca principalmente a la enseñanza académica?" resalta la categoría "casi siempre" con 67\%. Sin embargo muestra valores similares en los ítems "Has encontrado el curso formativamente estimulante" y "¿Las actividades son direccionadas en aprendizaje social y emocional para desarrollar las habilidades blandas?"

Las ultimas 2 dimensiones no suelen presentar un comportamiento similar, sino que los valores se distribuyen en todas las categorías. Por tanto las dimensiones más representativas son las dimensiones 1 y 2.

\section{Conclusión}

Se analizó la estructura del cuestionario realizando un análisis factorial para determinar la validez y confiabilidad del instrumento (cuestionario) en cada uno de las dimensiones con sus respectivos ítems. Las pruebas a que fueron sometidas resultaron satisfactorias, por lo que se recomienda su utilización en investigaciones futuras relacionadas a las variables como el enfoque de la educación virtual y al aprendizaje holístico.

De acuerdo a la investigación queda demostrado que el enfoque de la educación virtual cumple con impartir un aprendizaje holístico, que se optó frente a la Pandemia del COVID 19 en estudiantes universitarios, tal como muestra la hipótesis planteada al inicio del estudio y las tablas elaboradas en torno al tema. Aun no existen investigaciones similares ni parecidas al estudio.

En la educación digital, de acuerdo a la experiencia empírica, es necesario planificar las acciones que se va desarrollar, durante las clases no presenciales (el contenido, interacción, actividades de aprendizaje, evaluación y las herramientas tecnológicas), con el fin de impulsar un aprendizaje activo, donde el estudiante se convierte en constructor de su propio aprendizaje (genere ideas, discute, argumenta, gestiona aprendizaje con autonomía, construye, crea, genera conocimiento, soluciona problemas, etc.). Para ello es imprescindible tender redes de comunicación (sincrónica, asincrónica), entre el docente y estudiante, así como también, estar en constante renovación con las estrategias y técnicas actuales.

El objetivo a futuro es impulsar un espacio de aprendizaje seguro, activo con las herramientas digitales adecuadas que incorporé al estudiante a la investigación (buscar 
conocimientos basado en la innovación, formular opiniones con ideas originales, promover el pensamiento divergente y asunción de riesgos), a la responsabilidad social (Impulsar el autocontrol, la actitud ética, proactiva, y visión inclusiva), y al liderazgo (liderar equipos disciplinarios o multidisciplinarios, demostrar empatía, respeto, etc.), con la intención de prepararlos, para enfrentar a un mundo globalizado, que con pasos agigantados incorpora al ser humano a la competitividad y a la meritocracia. Asimismo, el compromiso de las autoridades de los estamentos universitarios, deben garantizar la capacitación en competencias virtuales a los docentes, para que puedan convertirse en residentes virtuales y proporcionar a los estudiantes una excelencia formación académica. 


\section{Agradecimiento}

Agradecimiento especial a los estudiantes de la Universidad Nacional Mayor de San Marcos y de la Universidad Nacional Federico Villarreal quienes con sus ideas dieron luces a la presente investigación.

\section{Referencias}

Abreu, J. (2020) Tiempos de coronavirus: la educación en línea como respuesta a la crisis, daena: International Journal of Good Conscience. http://www.spentamexico.org/v15n1/A1.15(1)1-15.pdf/

Allen, E. Seaman, J. (2017) Digital compass learning: distance education enrollment report 2017. https://eric.ed.gov/?id=ED580868/

Briceño, Jesús, Cañizales, Benito, Rivas, Yasmelis, Lobo, Hebert, Moreno, Emilia,

Velásquez, Iván y Ruzza, Ivone. (2010).La holística y su articulación con la generación de teorías. http://www.redalyc.org/articulo.oa?id=35616720008/

Cardini, A., Bergamaschi, A., D“Alessandre, V., Torre, E., Ollivier, A., (2020) Educar en pandemia: entre el aislamiento y la distancia socia. file:///C:/Users/tottus/Downloads/Educar-en-pandemia-Entre-el-aislamiento-y-ladistancia-social.pdf

CEPAL-UNESCO. (2020) la educación en tiempos de la pandemia de COVID-19. https://drive.google.com/file/d/1a2GqbUY6RTriUC1gxiOiwUYZpYq382zj/view/

Cubas, M. (2013). Causas de la escasez de talento en el Perú/ Entrevistado, portal financiero de canal N. https://www.youtube.com/watch?v=L41rX1RckOw

Durall, E., Gros, B., Maina, M., Johnson, L. \& Adams, S. (2012). Perspectivas tecnológicas: educación superior en Iberoamérica 2012-2017. Austin, Texas: The New Media Consortium.

Gallego, J. (2013) Educación a distancia y virtual: enfoque conceptual y metodológico en la Corporación Universitaria. file:///C:/Users/tottus/Downloads/Dialnet-EducacionADistanciaYVirtual7083558.pdf

Gallegos, R., (1999). Educación holista: Pedagogía del amor universal. México, Editorial PAX.

Gluyas, I., Esparza, R., Romero, R. y Rubio J. (2015) Modelo de educación holística: Una propuesta para la formación del ser humano. file:///C:/Users/tottus/Downloads/Modelo de educacion holistica Una propuesta para $1 \% 20(2)$.pdf./

Hernández S., Fernández, C. y Baptista, P. (2010). Metodología de la investigación. (Quinta Edición). México. McGraw-Hill.

López, C., (2018) La Educación Holística desde una Perspectiva Humanista. file:///C:/Users/tottus/Downloads/CsarEnriqueLpezArrillaga.pdf/ 
Rodríguez, M. (2010) Métodos de investigación, diseño de proyectos y desarrollo de tesis en ciencias administrativas, organizacionales y sociales. Universidad Autónoma de Sinaloa México.

Sotelo, M. (2017) Mentor tic. Lima Perú, Editorial San Marcos E. I. R. L.

UNESCO. (2020). Education inthe time of COVID -19. https://unesdoc.unesco.org/search/NEXPLORE-8ca029fa-ddba-4e57-85c0-18d3810cc961

Velazque L., Valenzuela, C., Salazar, F. y Lloyd, M. (2020) Desigualdades educativas y la brecha digital en tiempos de pandemia COVID-19. Repercusiones en la educación universitaria.

https://revistasinvestigacion.unmsm.edu.pe/index.php/odont/article/view/17766 /14848/ 


\section{Autores}

DINA INGA-LINDO, obtuvo su grado de Doctora en Ciencias de la educación por la Universidad Nacional Enrique Guzmán y Valle (Perú) en 2019. Obtuvo el grado de Magister en Investigación y Docencia en la Universidad Nacional Pedro Ruiz Gallo de Lambayeque (Perú) en 2011. Obtuvo el título de Licenciada en Pedagogía y Humanidades. Especialidad Español y Literatura, en la universidad Nacional del Centro del Perú, en 2002

Actualmente es docente en la Universidad Nacional Mayor de San Marcos y docente en la Universidad Nacional Federico Villarreal. Coordinadora en el área de Ciencias Económicas y de la Gestión en la Universidad Nacional Mayor de San Marcos. ha sido directora de Estudios Generales de la Universidad Peruana de Integración Global, Coordinadora Académica de la Facultad de Derecho y Ciencias Políticas, Es autora de diversos libros y poemarios. Sus principales temas de investigación; investigación cuantitativa, técnicas y estrategias de enseñanza - aprendizaje, Enfoques educativos, Tecnología Educativa, Innovación Pedagógica, Gestión Educativa. Últimos artículos publicados en diversas revistas indexadas a nivel nacional e internacional.

FELIPE AGUIRRE-CHÁVEZ, Doctor en Educación por la Universidad Nacional de Educación del Perú (2018), Master en Educación de la Creatividad por la universidad Cátedra UNESCO de Cuba. (2008). Realizó estudios de Ciencias Sociales y es Licenciado en Educación por la Universidad Champagnat de Perú (2000).

Actualmente es docente ordinario de la Universidad Marcelino Champagnat y docente ordinario de la Universidad Nacional Mayor de San Marcos. Participa como docente invitado en la Escuela de Posgrado del Centro de Altos Estudios Nacionales (CAEN). Docente de la Escuela de Doctoral de la Universidad Nacional de Educación. Docente de la Escuela Doctoral de la Universidad Nacional de Huancavelica Docente de la Escuela de Posgrado de la Universidad Nacional Hermilio Validizán de Huánuco. 